\title{
Expression of ATP transporters is associated with histologic regression of rectal carcinoma after neoadjuvant chemoradiotherapy
}

\author{
Duck-Woo Kim ${ }^{1 \#}$, Hyun-Chul Kim ${ }^{2 \#}$, Bo Hyung Kim³ ${ }^{3}$ Myung Jo Kim ${ }^{4}$ Eun Shin ${ }^{2,5}$ \\ ${ }^{1}$ Department of Surgery, Seoul National University Bundang Hospital, Seongnam, South Korea; ${ }^{2}$ Department of Pathology, Hallym University \\ Dongtan Sacred Heart Hospital, Hwaseong, South Korea; ${ }^{3}$ Department of Clinical Pharmacology and Therapeutics, Kyung Hee University \\ Hospital, Kyung Hee University College of Medicine, Seoul, South Korea; ${ }^{4}$ Deparment of Surgery, Chungbuk National University Hospital, \\ Cheongju, South Korea; ${ }^{5}$ Department of Pathology, Seoul National University Bundang Hospital, Seongnam, South Korea \\ Contributions: (I) Conception and design: BH Kim, E Shin; (II) Administrative support: MJ Kim; (III) Provision of study materials or patients: DW \\ Kim; (IV) Collection and assembly of data: MJ Kim; (V) Data analysis and interpretation: DW Kim, HC Kim, E Shin; (VI) Manuscript writing: All \\ authors; (VII) Final approval of manuscript: All authors. \\ \#These authors contributed equally as first authors to this work. \\ Correspondence to: Eun Shin. Department of Pathology, Hallym University Dongtan Sacred Heart Hospital, Hwaseong 18450, South Korea. \\ Email: sea4197@gmail.com.
}

Background: Preoperative chemoradiotherapy (CRT) has been established as a treatment of choice for locally advanced rectal cancer. However, the responses to CRT range very widely and there is no specific molecular marker definitively proven to be predictive of responses to CRT. We aimed to investigate the relationship between the expression of ATP-binding cassette (ABC) transporters and cancer stem cell (CSC) markers in pretreatment biopsy samples and pathological response to CRT in rectal cancer.

Methods: The immunohistochemical expression of three ABC transporters (ABCG2, ABCC2, and ABCC3) and two CSC markers (SOX2 and LGR5) was determined in 76 biopsy specimens from rectal cancer patients who underwent preoperative CRT. The association between protein expression and pathologic tumor regression grade was statistically analyzed.

Results: Fifty-eight (76.3\%) cases were classified as chemoradio-resistant group and 18 (23.7\%) cases as chemoradio-sensitive group and pathological complete remission was found in $8(10.5 \%)$ cases. ABCG2 was frequently expressed in chemoradio-sensitive group $(\mathrm{P}=0.042)$, while expression of ABCC2 was found in chemoradio-resistant group $(\mathrm{P}=0.014)$. Low expression of ABCC2 was associated with pathologic complete remission $(\mathrm{P}=0.008)$.

Conclusions: Immunohistochemical expression of ABCC2 and ABCG2 was associated with tumor regression after preoperative $\mathrm{CRT}$ in rectal cancer. In particular, $\mathrm{ABCC} 2$ was associated with resistance to CRT of rectal cancer. These markers might be used as biomarkers for predicting response against CRT in rectal cancer.

Keywords: ABC transporter; cancer stem cell (CSC); chemoradiotherapy; colorectal cancer; immunohistochemistry

Submitted Sep 19, 2018. Accepted for publication Dec 04, 2018.

doi: $10.21037 /$ tcr.2018.12.15

View this article at: http://dx.doi.org/10.21037/tcr.2018.12.15

\section{Introduction}

Patients with rectal cancers are known to have an increased rate of local recurrence and decreased survival time compared to patients with tumors of the upper colon. This is due primarily to the surgical constraints imposed by the location of the rectum within the pelvis. 
Preoperative chemoradiotherapy (CRT) has been established as a treatment of choice for locally advanced rectal cancer after the 1990s. Compared with postoperative CRT, the preoperative approach was superior in terms of overall compliance rate, reduced toxicity, superior rate of local control, and sphincter preservation (1). Moreover, favored long-term outcomes have been reported in patients with good CRT responses and in particular, pathological complete response (pCR) is known to be associated with excellent prognostic outcome $(2,3)$. Because of these advantages, preoperative CRT has become the preferred treatment for patients with stage II or III rectal cancer.

However, preoperative CRT is not equally beneficial for all patients with rectal cancer. Some patients display minimal response to CRT, whereas others show pCR. Patients expected to be poor responders should undergo alternative treatments early in the course of disease, thus also avoiding frequent CRT-related side effects such as enteritis, anorexia, thromboembolic events, and radiation dermatitis (4). On the other hand, prompt and clear-cut recognition of pCR patients could spare them unneeded surgical intervention, along with related functional consequences. Thus, prediction of response to CRT is very important for choosing the proper treatment option and avoiding unnecessary adverse effects and raising of costs.

Clinicopathological and radiological features have limited ability to predict response to preoperative CRT because of lack of sensitivity and specificity (5-7). Therefore, a wide variety of genetic and molecular biomarkers with the potential to predict the response have been investigated. For example, p53, epidermal growth factor receptor, vascular endothelial growth factor, thymidylate synthase, Ki-67, p21, Bcl-2, and Bax have all been reported to be associated with therapeutic response to preoperative CRT (8-10). However, the results have been controversial and inconclusive, and no specific molecular marker has yet been definitively proven to be predictive of response to CRT, so none have currently reached the clinic $(5,6)$.

Cancer stem cells (CSCs) represent a small population of cancer cells, sharing common properties with normal stem cells such as the ability of self-renewal and multidirectional differentiation. Because of these properties, after undergoing radio or chemotherapy, the residual CSCs can rapidly proliferate to re-establish the tumor. In addition, CSCs can survive during anti-cancer treatment though various drug resistance mechanisms including the upregulation of drug efflux transporters, represented by ATPbinding cassette (ABC) transporters (11-13). Therefore, one can assume that the expression of CSC markers or $\mathrm{ABC}$ transporters might be associated with resistance to anticancer treatment including chemotherapy or CRT.

In this study, we performed a pilot investigation to examine the relationship between the expression of $\mathrm{ABC}$ transporters and CSC markers in pretreatment biopsy samples and pathological response to CRT by postoperative histological examination of rectal cancer patients who received preoperative CRT.

\section{Methods}

\section{Patients}

A total of 76 patients with locally advanced rectal carcinoma were treated with preoperative CRT and surgical resection at the Department of Surgery, Seoul National University Bundang Hospital between May 2006 and December 2014. Histopathologic and clinical data were obtained from the medical records and pathologic reports of the patients. All patients were treated with preoperative CRT. The total dose of radiation in most cases was 45 Gy within 6 weeks, usually 1.5 Gy per treatment, five times per week. The total dose range was 40-50 Gy. Capecitabine- or 5-fluorouracil (5-FU)-based chemotherapeutic regimens were used in the patients with radiation therapy: capecitabine in 50 patients, $5-\mathrm{FU}$ and leucovorin in 25 patients, and 5-FU alone in one patient. Pathologic stage was determined according to the grading system of the 8th edition of the American Joint Committee on Cancer. Informed consent from the patients and approval of the local ethics committee were obtained.

\section{Classification of response to preoperative CRT}

Tumor response to preoperative CRT was evaluated pathologically in postoperative specimens according to the Dworak regression grading system. According to these standards, the therapeutic effect was categorized into five grades: tumor regression grade (TRG) 0, no regression; TRG 1, dominant tumor mass with obvious fibrosis and/ or vasculopathy; TRG 2, dominant fibrotic changes with few tumor cells or groups (easy to find); TRG 3, very few (difficult to find microscopically) tumor cells in fibrotic tissue with or without mucous substance; TRG 4, no tumor cells, only fibrotic mass (total regression). TRG 0, TRG 1 and TRG 2 were combined to form the chemoradioresistant group $(58 / 76 ; 76.3 \%)$ in comparison with the chemoradio-sensitive group (TRG 3 and TRG 4; 18/76; 

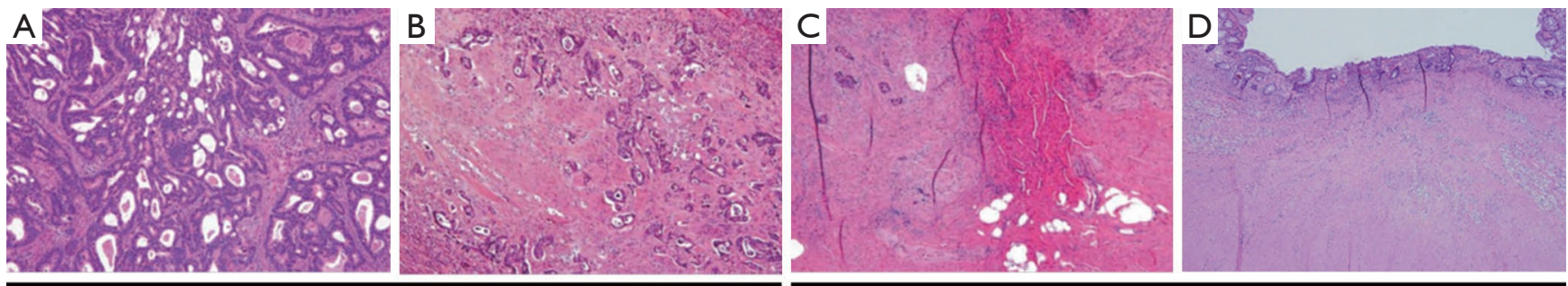

Chemoradio-resistant group

Chemoradio-sensitive group

Figure 1 Representative photographs of rectal cancers after neoadjuvant chemoradiation therapy. Tumor regression grade 1 (A), grade 2 (B), grade 3 (C), and grade 4 (D) by the Dworak regression grading system (Hematoxylin \& Eosin stain, $\times 200$ ).

$23.7 \%)$. Representative photographs for each tumor regression grade are presented in Figure 1.

\section{Immunobistochemistry}

Five biomarkers were chosen as candidate predictive factors for the efficacy of preoperative CRT. These factors included three $\mathrm{ABC}$ transporters, namely $\mathrm{ABCG} 2, \mathrm{ABCC} 2$, and $\mathrm{ABCC} 3$, and two stem cell markers, namely leucinerich repeat-containing $G$ protein-coupled receptor 5 (LGR5) and sex-determining region Y-box 2 (SOX2). Immunohistochemical staining was performed using a BenchMark XT automated immunostaining system (Ventana Medical System, Tucson, AZ). Briefly, 4- $\mu$ m-thick sections were cut from paraffin tissue blocks, mounted on positively charged slides, and dried at $62{ }^{\circ} \mathrm{C}$ for $30 \mathrm{~min}$. After undergoing heat epitope retrieval for $60 \mathrm{~min}$ in ethylenediaminetetraacetic acid ( $\mathrm{pH}$ 8.0) in an autostainer, the samples were incubated with individual primary antibodies and subsequently treated using the UltraView Universal DAB kit (Ventana Medical Systems, Tucson, AZ) for visualization of staining. The following antibodies were used: ABCG2 (polyclonal, 1:200; Alexis Biochemicals); ABCC2 (clone M2 III-6, 1:50; Abcam); ABCC3 (clone M3II-9, 1:20; Abcam); LGR5 (polyclonal, 1:100; SigmaAldrich), SOX2 (clone 6F 1.2, 1:500; Millipore). The intensities of cytoplasmic or membranous staining were semi-quantitatively measured at a $200 \times$ magnification, and staining intensity was categorized as negative (score $=0$ ), weak $($ score $=1)$, moderate $($ score $=2)$, or strong $($ score $=3$ ). The percentage of immunoreactive cells was also assessed. Because there are no absolute criteria for the examined proteins, by testing a series of different values, the proteins were considered to be overexpressed when $>10 \%$ of tumor cells had an intensity score of $\geq 1$.

\section{Statistical analysis}

Statistical analysis was performed using the SPSS version 21.0 software package (SPSS, Chicago, IL, USA). The correlations between the status of protein expression and the clinicopathological features including tumor regression grade were analyzed using Pearson's $\chi^{2}$ test and the Fisher's exact probability test. Multivariate logistic regression analysis was performed to determine independent parameters associated with the response to CRT with the backward stepwise selection procedures. For the data on analysis of survival, the differences between survival rates were determined using the log-rank test. All statistics were 2-sided and a value of $\mathrm{P}<0.05$ was considered statistically significant.

\section{Results}

\section{Patient and tumor characteristics}

There were 29 (38.2\%) females and 47 (61.8\%) males involved in the study. The mean age was 60.4 years (range of 31-86 years). Abdominoperineal resection was performed in $15(19.7 \%)$ patients and anal sphincter-preserving operation was performed in $59(77.6 \%)$ patients. Transanal local excision was performed in $2(2.6 \%)$ patients. Image studies using computed tomography or magnetic resonance imaging classified 4 cases as clinical $T$ stage 2, 67 cases as stage 3 , and 5 cases as stage 4 . Sixty-seven cases were expected to have lymph node metastasis, and distant metastasis was revealed in 5 cases by image equipment. After CRT and surgical treatment, $58(76.3 \%)$ cases were classified as chemoradio- 
Table 1 Clinicopathologic characteristics

\begin{tabular}{|c|c|}
\hline Characteristic & No. of patients $(n=76)$ \\
\hline Age (years), mean \pm SD [range] & $60.4 \pm 12.4[31-86]$ \\
\hline \multicolumn{2}{|l|}{ Gender, n (\%) } \\
\hline Female & $29(38.2)$ \\
\hline Male & $47(61.8)$ \\
\hline \multicolumn{2}{|l|}{ Tumor location, n (\%) } \\
\hline Upper (AV 10-15 cm) & $5(6.6)$ \\
\hline Middle (AV 6-9 cm) & $15(19.7)$ \\
\hline Lower (AV 1-5 cm) & $56(73.7)$ \\
\hline \multicolumn{2}{|l|}{ cT stage, n (\%) } \\
\hline 2 & $4(5.3)$ \\
\hline 3 & $67(88.2)$ \\
\hline 4 & $5(6.6)$ \\
\hline \multicolumn{2}{|l|}{ cN stage, n (\%) } \\
\hline 0 & $9(11.8)$ \\
\hline 1 & $29(38.2)$ \\
\hline 2 & $38(50.0)$ \\
\hline \multicolumn{2}{|l|}{ cM stage, n (\%) } \\
\hline 0 & $71(93.4)$ \\
\hline 1 & $5(6.6)$ \\
\hline \multicolumn{2}{|l|}{ Surgical procedures, n (\%) } \\
\hline Abdomioperineal resection & $15(19.7)$ \\
\hline Low anterior resection & $59(77.6)$ \\
\hline Transanal local excision & $2(2.6)$ \\
\hline \multicolumn{2}{|l|}{ Tumor regression grading, $\mathrm{n}(\%)$} \\
\hline 0 & $4(5.3)$ \\
\hline 1 & $14(18.4)$ \\
\hline 2 & $40(52.6)$ \\
\hline 3 & $10(13.2)$ \\
\hline 4 & $8(10.5)$ \\
\hline \multicolumn{2}{|l|}{ ypT stage, n (\%) } \\
\hline 0 & $8(10.5)$ \\
\hline In situ tumor & $3(3.9)$ \\
\hline 1 & $5(6.6)$ \\
\hline 2 & $17(22.4)$ \\
\hline 3 & $43(56.6)$ \\
\hline
\end{tabular}

Table 1 (continued)
Table 1 (continued)

\begin{tabular}{lc}
\hline Characteristic & No. of patients $(\mathrm{n}=76)$ \\
\hline $\mathrm{ypN}$ stage, $\mathrm{n}(\%)$ & $46(62.2)$ \\
0 & $26(35.1)$ \\
1 & $2(2.7)$ \\
2 & \\
Vascular invasion, $\mathrm{n}(\%)$ & $68(89.5)$ \\
Not identified & $8(10.5)$ \\
Present & \\
Lymphatic invasion, $\mathrm{n}(\%)$ & $65(85.5)$ \\
Not identified & $11(14.5)$ \\
Present & \\
Perineural invasion, $\mathrm{n}(\%)$ & $60(78.9)$ \\
Not identified & $16(21.1)$ \\
Present & \\
Disease progression after & \\
operation, $\mathrm{n}$ (\%) & $61(80.3)$ \\
No & \\
Yes & \\
\hline
\end{tabular}

resistant group and $18(23.7 \%)$ cases as chemoradiosensitive group. pCR was found in 8 (10.5\%) cases. Lymph node metastasis was assessed in 74 cases and observed in $28(37.8 \%)$ cases. Vascular, lymphatic and perineural invasion were found in $10.5 \%, 14.5 \%$, and $21.1 \%$ of the cases, respectively. Fifteen patients (19.7\%) showed local recurrence or disease progression after treatment. Table 1 shows the clinical and pathologic characteristics of the 76 rectal cancer cases.

\section{Expression of CSC markers and ABC transporters}

ABCG2, ABCC2, and ABCC3 were expressed in $52(68.4 \%), 56(73.7 \%)$, and $9(11.8 \%)$ out of 76 pretreatment rectal cancer specimens, respectively. The expression rate of SOX2 was $82.9 \%$ (63/76). LGR5 expression was assessed in 72 cases and the rate was $95.8 \%$ (69/72). Representative photographs of ABC transporter and CSC marker expression in rectal cancers are shown in Figure 2. The expression of proteins showed no correlation with clinical or pathologic parameters (data not shown). 

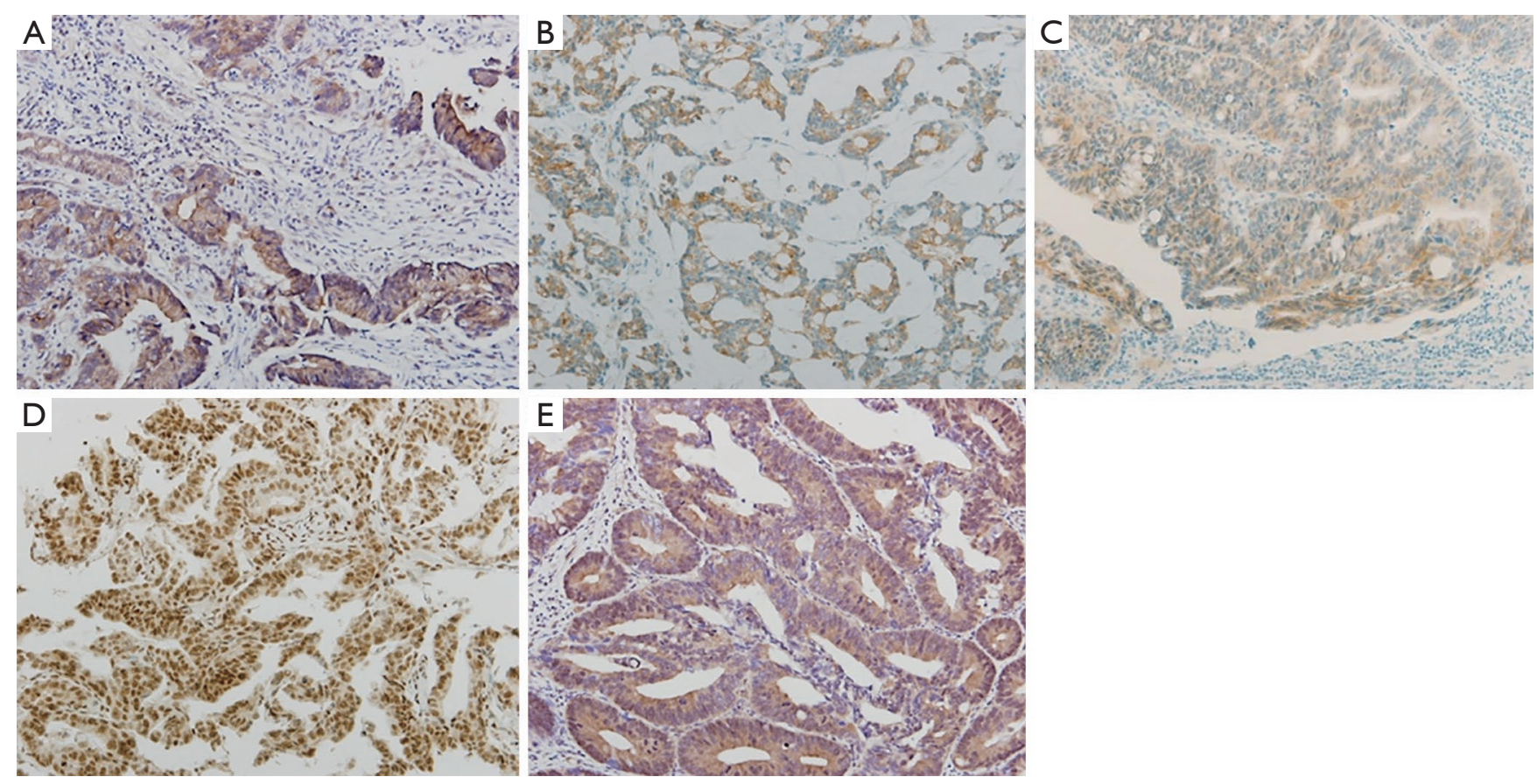

Figure 2 Immunohistochemical expression of ABC transporters and CSC markers in pretreatment biopsy samples of rectal cancer patients. Positive staining for ABCG2 (A), ABCC2 (B), ABCC3 (C), SOX2 (D), and LGR5 (E) (Immunohistochemical stain, ×200).

\section{Association between ABC transporters or CSC markers in pretreatment biopsy tissue and response to chemoradiotherapy}

The expression of ABCG2 was frequently observed in chemoradio-sensitive group $(\mathrm{P}=0.042)$, while the $\mathrm{ABCC} 2$ was frequently expressed in chemoradio-resistant group $(\mathrm{P}=0.014)$. Other clinical parameters including age, gender, tumor location, and clinical TNM stage showed no significant association (Table 2). Multivariate analysis using protein expression and pretreatment clinical parameters such as sex, age, tumor location, and clinical TNM stage, indicated that the expression of the two proteins was independently associated with response to chemoradiotherapy $(\mathrm{P}=0.029$ and 0.008 , respectively) (Table $S 1$ ).

\section{Association between ABC transporters or CSC markers in pretreatment biopsy tissue and pathologic complete remission}

Among the 56 cases with positivity for ABCC2, only 2 cases (3.6\%) showed pCR, whereas 6 out of 20 cases (30\%) without ABCC2 expression showed pCR. This difference was statistically significant $(\mathrm{P}=0.003)$. The expression of other proteins showed no correlation with pCR. Among clinical parameters, lower clinical $\mathrm{T}$ stage was associated with higher rates of pCR (Table 3). Multivariate analysis was performed using protein expression and pretreatment parameters including sex, age, tumor location, and clinical TNM stage, and only ABCC2 expression showed statistically significant association with complete tumor regression $(\mathrm{P}=0.008)$ (Table $S 2)$.

\section{Disease-free survival analysis}

Perineural invasion and presence of metastasis in more than one lymph node had a significant association with shorter disease free survival ( $\mathrm{P}=0.001$ and 0.011 , respectively). Cases with chemoradio-resistance or expression of ABCC2 showed tendency for shorter disease free survival than cases with chemoradio-sensitivity, however statistical significance was not found ( $\mathrm{P}=0.130$ and 0.246 , respectively).

\section{Discussion}

In the present study, we explored the suitability of $\mathrm{ABC}$ transporters and CSC markers as predictive biomarkers of response to neoadjuvant CRT in patients with rectal cancer. We found an association of both ABCC2 and ABCG2 with tumor response to CRT. Specifically, ABCC2 expression 
Table 2 Chemoradio-sensitivity according to preoperative characteristics and expression of $\mathrm{ABC}$ transporters and cancer stem cell markers

\begin{tabular}{lccc}
\hline Characteristics & $\begin{array}{c}\text { Chemoradio- } \\
\text { resistant group }\end{array}$ & $\begin{array}{c}\text { Chemoradio- } \\
\text { sensitive group }\end{array}$ & $\begin{array}{c}\mathrm{P} \\
\text { value }\end{array}$ \\
\hline Age (years), $\mathrm{n}(\%)$ & & & 0.574 \\
$\leq 60$ & $30(51.7)$ & $7(38.9)$ & \\
$>60$ & $28(48.3)$ & $11(61.1)$ &
\end{tabular}

Gender, n (\%)

Female

Male

$23(39.7)$

0.783

$35(60.3)$

$6(33.3)$

$12(66.7)$

Tumor location, n (\%)

Upper

Middle

Lower

cT stage, $\mathrm{n}(\%)$

2

3

4

cN stage, n (\%)

0

1

2

cM stage, n (\%)

0

1

ABCG2, n (\%)

Negative

Positive

ABCC2, n (\%)

Negative

Positive

ABCC3, n (\%)

Negative

Positive

SOX2, n (\%)

Negative

Positive

LGR5, n (\%)

Negative

Positive
2 (12.5)

14 (87.5)

$\begin{array}{ll}1(1.8) & 2(12.5) \\ 55(98.2) & 14(87.5)\end{array}$

3 (5.2) $\quad 2(11.1)$

10 (17.2)

5 (27.8)

45 (77.6)

$11(61.1)$

2 (3.4)

51 (87.9)

5 (8.6)

7 (12.10)

20 (34.5)

31 (53.4)

$2(11.1)$

16 (88.9)

$0(0.0)$

0.482

2 (11.1)

9 (50.0)

7 (38.9)

0.365

$(2.20$

54 (93.1)

4 (6.9)

17 (94.4)

$1(5.6)$

0.214

(1)

- $-2-2$

cN stage

0

1

2

cM stage

0.841

0.841

0.042

22 (37.9)

2 (11.1)

36 (62.1)

16 (88.9)

11 (19.0)

47 (81.0)

9 (50.0)

1.000

16 (88.9)

51 (87.9)

7 (12.1)

2 (11.1)

1.000

0.014

0

1

ABCG2

Negative

Positive

$\mathrm{ABCC} 2$

Negative

Positive

ABCC3

Negative

Positive

SOX2

1.000

10 (17.2)

3 (16.7)

$48(82.8)$

$15(83.3)$

0.122

Negative

Positive

LGR5
Table 3 Complete remission rate according to preoperative characteristics and expression of $\mathrm{ABC}$ transporters and cancer stem cell markers

Age (years)

Complete remission (\%) $\mathrm{P}$ value

0.256

2/36 (5.6)

$6 / 39(15.4)$

0.703

$2 / 29(6.9)$

$6 / 47(12.8)$

0.686

$1 / 5(20.0)$

$2 / 15(13.3)$

$5 / 56(8.9)$

0.025

2/4 (50.0)

$6 / 67(9.0)$

$0 / 5(0.0)$

0.500

$0 / 9(0.0)$

$4 / 29(13.8)$

$4 / 38(10.5)$

0.735

$7 / 71(9.9)$

$1 / 5(20.0)$

1.000

2/24 (8.3)

$6 / 52(11.5)$

0.003

$6 / 20(30.0)$

2/56 (3.6)

0.585

8/67 (11.9)

$0 / 9(0.0)$

$2 / 13(15.4)$

$6 / 63(9.5)$

0.233

$\begin{array}{ll}\text { Negative } & 1 / 3(33.3) \\ \text { Positive } & 5 / 69(6.7)\end{array}$


was higher in CRT-resistant tumors, whereas ABCG2 was more highly expressed in CRT-sensitive tumors.

Cell-based evidence for a role of ABC transporters in cancer resistance to CRT has been previously reported (14-16). Moreover, such a relationship has been found in breast cancer and esophageal adenocarcinoma (14,17-21). Furthermore, in colorectal cancer, clinically relevant transcriptional variations have been reported for various ABC transporters (22). In rectal cancer, association between resistance to CRT and ABCC3 was revealed by using patients' samples and in vitro experiment (23). Immunohistochemical expression of ABCC4 was found associated with resistance of rectal cancer to CRT and single nucleotide polymorphism of ABCC4 also showed association with response to CRT in rectal cancer $(24,25)$.

We found that ABCC2 is highly expressed in the CRTresistant group, while CRT responders displayed relatively low ABCC2 expression. ABCC2 is known to confer resistance to a number of different anti-cancer drugs (15). ABCC2 positivity has been associated with chemoresistance of esophageal cancer (20) and with resistance to CRT in head and neck squamous cell carcinoma (26). Moreover, an in vitro study showed that $\mathrm{ABCC} 2$ is involved in drug resistance of colorectal cancer cell lines (27). On the other hand, a study addressing possible ABCC2-related drug resistance mechanisms in 5-FU-treated patients with colorectal cancer led to inconclusive results (28). Notably, a specific association between $\mathrm{ABCC} 2$ expression and the response to CRT of rectal cancer has not yet been investigated and, to our knowledge, our study is the first to report such a relationship. Our findings support the suitability of ABCC2 as a biomarker for the early identification of patients with poor response to neoadjuvant CRT, and who will most likely benefit from alternative treatments.

The expression of ABCG2 was previously shown to be associated with the response to 5-FU-based chemotherapy in breast cancer and metastatic colorectal cancer $(19,29)$. We also found a relationship between ABCG2 expression and the response of rectal tumors to CRT. Notably, CRT-sensitive rectal tumors displayed a higher ABCG2 expression, compared to the CRT-resistant tumors. A similar correlation has been previously observed with another $\mathrm{ABC}$ transporter, $\mathrm{ABCC} 1$, the expression of which was found to be higher in responders to neoadjuvant chemotherapy, in esophageal adenocarcinoma (21). These results may reflect differences related to the specific type of anti-cancer treatment employed and kind of tumor.

The expression of two other proteins, the CSC markers
SOX2 and LGR5, was also investigated in relation to CRT response in rectal cancer. SOX2 is known as a transcription factor essential for the maintenance of pluripotent stem cells (30) and LGR5, a cell-surface molecule involved in signaling, is considered a global marker of adult stem cells (31). A role of these two proteins in the response of large bowel cancer to chemotherapy has been previously reported. Transcription of SOX2 in residual rectal cancer cells after CRT was found to be significantly associated with poor survival (32), and metastatic colorectal cancer patients with low LGR5 levels showed better response to 5-FU-based treatment (33). However, in our rectal cancer patients, SOX2 and LGR5 expression was not associated with response to neoadjuvant CRT.

We believe that our study provides important information about the role of ABC transporters as biomarkers of CRT response in rectal cancer. However, one major limitation of our study is the relative small number of enrolled cases. Additional studies with larger numbers of will be necessary to ascertain the applicability of these markers in clinical routine for the prediction of rectal tumor response to CRT.

\section{Conclusions}

Immunohistochemical evaluation showed that ABCC2 and ABCG2 expression was associated with tumor response to preoperative CRT in rectal cancer. Based on our results, we propose these two proteins as potential biomarkers of response to CRT in rectal cancer.

\section{Acknowledgments}

Funding: This work is supported by the Seoul National University Bundang Hospital research fund grant (No. 14-2015-009).

\section{Footnote}

Conflicts of Interest: All authors have completed the ICMJE uniform disclosure form (available at http://dx.doi. org/10.21037/tcr.2018.12.15). The authors have no conflicts of interest to declare.

Ethical Statement: The authors are accountable for all aspects of the work in ensuring that questions related to the accuracy or integrity of any part of the work are appropriately investigated and resolved. The study was conducted in accordance with the Declaration of Helsinki 
(as revised in 2013). Informed consent was obtained from the patients. The study was conducted in accordance with the Declaration of Helsinki (as revised in 2013). The study was reviewed and approved by the Ethics Committees of the Seoul National University Bundang Hospital (B1512$326 / 302)$.

Open Access Statement: This is an Open Access article distributed in accordance with the Creative Commons Attribution-NonCommercial-NoDerivs 4.0 International License (CC BY-NC-ND 4.0), which permits the noncommercial replication and distribution of the article with the strict proviso that no changes or edits are made and the original work is properly cited (including links to both the formal publication through the relevant DOI and the license). See: https://creativecommons.org/licenses/by-nc-nd/4.0/.

\section{References}

1. Sauer R, Liersch T, Merkel S, et al. Preoperative versus postoperative chemoradiotherapy for locally advanced rectal cancer: results of the German CAO/ARO/AIO-94 randomized phase III trial after a median follow-up of 11 years. J Clin Oncol 2012;30:1926-33.

2. Yeo SG, Kim DY, Kim TH, et al. Pathologic complete response of primary tumor following preoperative chemoradiotherapy for locally advanced rectal cancer: long-term outcomes and prognostic significance of pathologic nodal status (KROG 09-01). Ann Surg 2010;252:998-1004.

3. Rödel C, Martus P, Papadoupolos T, et al. Prognostic significance of tumor regression after preoperative chemoradiotherapy for rectal cancer. J Clin Oncol 2005;23:8688-96.

4. Calvo FA, Serrano FJ, Diaz-Gonzalez JA, et al. Improved incidence of $\mathrm{pT} 0$ downstaged surgical specimens in locally advanced rectal cancer (LARC) treated with induction oxaliplatin plus 5-fluorouracil and preoperative chemoradiation. Ann Oncol 2006;17:1103-10.

5. Ryan JE, Warrier SK, Lynch AC, et al. Predicting pathological complete response to neoadjuvant chemoradiotherapy in locally advanced rectal cancer: a systematic review. Colorectal Dis 2016;18:234-46.

6. Dayde D, Tanaka I, Jain R, et al. Predictive and Prognostic Molecular Biomarkers for Response to Neoadjuvant Chemoradiation in Rectal Cancer. Int J Mol Sci 2017;18.

7. Santos MD, Silva C, Rocha A, et al. Predictive Response Value of Pre- and Postchemoradiotherapy Variables in
Rectal Cancer: An Analysis of Histological Data. Patholog Res Int 2016;2016:2164609.

8. Kuremsky JG, Tepper JE, McLeod HL. Biomarkers for response to neoadjuvant chemoradiation for rectal cancer. Int J Radiat Oncol Biol Phys 2009;74:673-88.

9. Miura K, Fujibuchi W, Ishida K, et al. Inhibitor of apoptosis protein family as diagnostic markers and therapeutic targets of colorectal cancer. Surg Today 2011;41:175-82.

10. Santos MD, Silva C, Rocha A, et al. Predictive clinical model of tumor response after chemoradiation in rectal cancer. Oncotarget 2017;8:58133-51.

11. Crea F, Danesi R and Farrar WL. Cancer stem cell epigenetics and chemoresistance. Epigenomics 2009;1:63-79.

12. Vinogradov $S$ and Wei X. Cancer stem cells and drug resistance: the potential of nanomedicine. Nanomedicine (Lond) 2012;7:597-615.

13. Fábián A, Barok M, Vereb G, et al. Die hard: are cancer stem cells the Bruce Willises of tumor biology? Cytometry A 2009;75:67-74.

14. Gillet JP, Efferth T and Remacle J. Chemotherapyinduced resistance by ATP-binding cassette transporter genes. Biochim Biophys Acta 2007;1775:237-62.

15. Nies AT, Magdy T, Schwab M, et al. Role of ABC transporters in fluoropyrimidine-based chemotherapy response. Adv Cancer Res 2015;125:217-43.

16. Di Nicolantonio F, Mercer SJ, Knight LA, et al. Cancer cell adaptation to chemotherapy. BMC Cancer 2005;5:78.

17. Schneider J, Gonzalez-Roces S, Pollan M, et al. Expression of LRP and MDR1 in locally advanced breast cancer predicts axillary node invasion at the time of rescue mastectomy after induction chemotherapy. Breast Cancer Res 2001;3:183-91.

18. Nooter K, Brutel de la Riviere G, Look MP, et al. The prognostic significance of expression of the multidrug resistance-associated protein (MRP) in primary breast cancer. Br J Cancer 1997;76:486-93.

19. Wang $M$, Wang $X$, Yuan J, et al. Expression of the breast cancer resistance protein and 5 -fluorouracil resistance in clinical breast cancer tissue specimens. Mol Clin Oncol 2013;1:853-7.

20. Yamasaki M, Makino T, Masuzawa T, et al. Role of multidrug resistance protein 2 (MRP2) in chemoresistance and clinical outcome in oesophageal squamous cell carcinoma. Br J Cancer 2011;104:707-13.

21. Langer R, Specht K, Becker K, et al. Association of pretherapeutic expression of chemotherapy-related genes 
with response to neoadjuvant chemotherapy in Barrett carcinoma. Clin Cancer Res 2005;11:7462-9.

22. Hlavata I, Mohelnikova-Duchonova B, Vaclavikova $\mathrm{R}$, et al. The role of $\mathrm{ABC}$ transporters in progression and clinical outcome of colorectal cancer. Mutagenesis 2012;27:187-96.

23. Yu Z, Zhang C, Wang H, et al. Multidrug resistance-associated protein 3 confers resistance to chemoradiotherapy for rectal cancer by regulating reactive oxygen species and caspase-3-dependent apoptotic pathway. Cancer Lett 2014;353:182-93.

24. Yu ZQ, Zhang C, Wang H, et al. Downregulation of ATP-binding cassette subfamily $\mathrm{C}$ member 4 increases sensitivity to neoadjuvant radiotherapy for locally advanced rectal carcinoma. Dis Colon Rectum 2013;56:600-8.

25. Santos MD, Silva C, Rocha A, et al. Prognostic and Therapeutic Potential Implications of Genetic Variability in Prostaglandin E2 Pathway Genes in Rectal Cancer. Anticancer Res 2017;37:281-91.

26. van den Broek GB, Wildeman M, Rasch CR, et al. Molecular markers predict outcome in squamous cell carcinoma of the head and neck after concomitant cisplatin-based chemoradiation. Int J Cancer 2009; 124:2643-50.

27. Shen K, Cui D, Sun L, et al. Inhibition of IGF-IR

Cite this article as: Kim DW, Kim HC, Kim BH, Kim MJ, Shin E. Expression of ATP transporters is associated with histologic regression of rectal carcinoma after neoadjuvant chemoradiotherapy. Transl Cancer Res 2018;7(6):1657-1665. doi: $10.21037 /$ tcr.2018.12.15 increases chemosensitivity in human colorectal cancer cells through MRP-2 promoter suppression. J Cell Biochem 2012;113:2086-97.

28. Mirakhorli M, Shayanfar N, Rahman SA, et al. Lack of association between expression of MRP2 and early relapse of colorectal cancer in patients receiving FOLFOX-4 chemotherapy. Oncol Lett 2012;4:893-7.

29. Lin PC, Lin HH, Lin JK, et al. Expression of ABCG2 associated with tumor response in metastatic colorectal cancer patients receiving first-line FOLFOX therapy-preliminary evidence. Int J Biol Markers 2013;28:182-6.

30. Takahashi K, Tanabe K, Ohnuki M, et al. Induction of pluripotent stem cells from adult human fibroblasts by defined factors. Cell 2007;131:861-72.

31. Nakata S, Phillips E and Goidts V. Emerging role for leucine-rich repeat-containing G-protein-coupled receptors LGR5 and LGR4 in cancer stem cells. Cancer Manag Res 2014;6:171-80.

32. Saigusa S, Tanaka K, Toiyama Y, et al. Correlation of CD133, OCT4, and SOX2 in rectal cancer and their association with distant recurrence after chemoradiotherapy. Ann Surg Oncol 2009;16:3488-98.

33. Hsu HC, Liu YS, Tseng KC, et al. Overexpression of Lgr5 correlates with resistance to 5-FU-based chemotherapy in colorectal cancer. Int J Colorectal Dis 2013;28:1535-46. 
Supplementary

Table S1 Multivariate analysis of correlation between expression of ABCG2 and ABCC2 and sensitivity to chemoradio-therapy

\begin{tabular}{lccccc}
\hline Characteristics & Coefficient & SE & Wald value & P value & HR \\
\hline ABCG2 & 1.847 & 0.846 & 4.769 & 0.029 & 0.158 \\
ABCC2 & -1.680 & 0.629 & 7.139 & 0.008 & 5.363 \\
\hline
\end{tabular}

SE, standard error; HR, hazard ratio.

Table S2 Multivariate analysis of correlation between expression of ABCG2 and ABCC2 and complete remission

\begin{tabular}{lccccc}
\hline Characteristics & Coefficient & SE & Wald value & P value & HR \\
\hline Clinical T stage & -2.319 & 1.284 & 3.263 & 0.071 & 0.098 \\
ABCC2 & -2.418 & 0.908 & 7.083 & 0.008 & 0.089 \\
\hline
\end{tabular}

SE, standard error; HR, hazard ratio. 